\title{
Sleep-related issues for recovery and performance in athletes
}

Invited review

Sarah Kölling ${ }^{1,2}$, Rob Duffield ${ }^{3}$, Daniel Erlacher ${ }^{4}$, Ranel Venter $^{2}, \&$ Shona L. Halson ${ }^{5}$

${ }^{1}$ Faculty of Sport Science, Ruhr-University Bochum, Bochum, Germany

${ }^{2}$ Department of Sport Science, Stellenbosch University, Stellenbosch, South Africa

${ }^{3}$ Sport \& Exercise Discipline Group, Faculty of Health, University of Technology Sydney, Sydney, Australia

${ }^{4}$ Institute of Sport Science, University of Bern, Bern, Switzerland

${ }^{5}$ Department of Physiology, Australian Institute of Sport, Canberra, Australia

Corresponding author

Sarah Kölling

Faculty of Sport Science, Ruhr-University Bochum, Bochum, Germany

Gesundheitscampus Nord 10

44801 Bochum, Germany

Telephone +492343225983

Fax +492343214245

Sarah.Koelling@,rub.de

Preferred running head: sleep-related issues in athletes

Abstract word count: 185

Text-only word count: 2467

Number of figures and tables: 0 


\begin{abstract}
The body of research that reports the relevance of sleep in high-performance sports is growing steadily. While the identification of sleep cycles and diagnosis of sleep disorders is limited to lab-based assessment via polysomnography, the development of activity-based devices estimating sleep patterns provides greater insight into the sleep behaviour of athletes in ecological settings. Overall, small sleep quantity and/or poor quality appears to exist in many athletic populations, though this may be related to training and competition context. Typical sleep-affecting factors are the scheduling of training sessions and competitions as well as impaired sleep-onset as a result of increased arousal prior to competition or due to the use of electronic devices before bedtime. Further challenges are travel demands which may be accompanied by jet-lag symptoms and disruption of sleep habits. Promotion of sleep may be approached via behavioural strategies, such as sleep hygiene, extending night-time sleep or daytime napping. Pharmacological interventions should be limited to clinically-induced treatments as evidence among healthy and athletic populations is lacking. As an aside, new evidence is emerging that dreams can be used to deliberately practice during sleep (lucid dreaming). How such practices impact on performance and recovery still requires further examination. To optimise and manage sleep in athletes, it is recommended to implement routine sleep monitoring on an individual basis.
\end{abstract}




\section{Introduction}

Sleep is increasingly gaining attention among sport scientists and practitioners as an important element to optimise sport performance and recovery. In fact, the critical importance of sleep's restorative effects in daily life makes it an integral part of the recovery processes for athletes. ${ }^{1}$ For example, the high physical and mental stress imposed as part of normal athlete training and competition routines requires appropriate recovery time to facilitate adaptive processes. Given the role of sleep in metabolic, hormonal and cognitive regeneration from daily activities when awake, ${ }^{2,3}$ appropriate sleep quality and quantity is of further importance for athletic populations. Moreover, changes in sleep behaviour enforced by training and competition create difficulties for athletes to obtain sufficient sleep to aid performance and recovery. ${ }^{4}$ Accordingly, the aim of this brief review is to summarise the role of sleep in athlete performance and recovery; particularly, factors affecting sleep behaviour, the prevalence of sleep loss among athletes, and recommendations for intervention strategies.

\section{Definition and measurement of sleep}

The sleep-wake continuum is regulated by the interaction of homeostatic mechanisms related to circadian processes, alongside external environmental cues which determine the time and duration of wakefulness and sleep periods. ${ }^{5}$ More specifically, these processes underlie endogenous functions, such as the thermoregulatory control of core body temperature; ${ }^{6}$ as well as exogenous mechanisms, including exposure to the daily light-dark cycle ${ }^{5}$ which is regulating the neurophysiology of sleep. In humans, sleep can be characterised based on two respective stages, including rapid eye movement (REM) and non-REM sleep (NREM sleep). ${ }^{7}$ In turn, NREM sleep is further categorised by three stages representing a depth continuum, with increasing arousal thresholds from stage N1 (light sleep) to N3 (deep sleep). In the course of the night, NREM and REM sleep alternate in cyclic fashion, with higher proportions of NREM in the first third of the night and expanding NREM episodes across the night. ${ }^{7}$

The measurement and classification of the respective stages of sleep are defined based on a collection of physiological characteristics determined from the gold-standard measures of polysomnography (PSG). These measures are derived from electroencephalogram, electromyogram, and electrooculogram measures ${ }^{7}$. Additional sensors to measure and diagnose sleep disorders include pulse oximetry, air flow and respiratory effort, electrocardiogram, leg movements, body temperature and position. PSG measures are utilised for clinical investigation of sleep cycles and to measure and diagnose symptoms of sleep disorders, such as sleep apnoea or restless leg syndrome. ${ }^{7}$ However, for routine assessments and regular monitoring of athletes, especially in high performance settings, PSG measures are intrusive, expensive and thus prohibitive given the competition and travel requirements of most athletes. Visiting a sleep laboratory for instance, and sleeping in an unfamiliar environment while being attached to numerous electrodes is not convenient or appropriate for most athletes - and somewhat counterintuitive to optimise recovery or competition preparation. Thus, this method indicates limited ecological validity and raises concerns regarding the accuracy of lab-based assessment to be representative of the 'normal' sleep habits in natural settings. Consequently, alternative methods include subjective sleep assessments and actigraphy. ${ }^{2}$ The development of sleep prediction algorithms from actigraphy-based measures has improved the accessibility of measurement in ecologically valid environments, and thus the reporting of sleep-wake behaviours among athletes in different field-based settings. ${ }^{8,9}$ Whilst practical, recent validity and reliability data further support their robustness for most sleep variables; yet some limitations do apply given that the underlying algorithms only represent estimations of sleep parameters and might differ considering the diversity of devices, misinterpretations, mishandling as well as technical errors. ${ }^{8,9,10}$ 


\section{Sleep in athletes}

Aside from physiological restoration and psychological well-being, memory consolidation is a function of sleep that is especially important for athletes in terms of learning procedural skills. ${ }^{11}$ This is supported by a recent review in which the effects of sleep loss on exercise performance, physiological responses to exercise and cognitive functions are analysed. ${ }^{3}$ Although there is proof that sleep deprivation ( $>24$ hours without sleep) reduces physical and cognitive functioning, it is not likely that total sleep loss is found in athletic populations. ${ }^{2}$ More apt (in a sport context) are situations of sleep disruption, where altered bed times or irregular waking result in reductions in sleep quantity or quality. Within these environments, the effect of 2-4 hours less sleep shows equivocal effects on physical performance or recovery (e.g., maximal physical efforts and gross motor performances may be maintained, while sport-specific performance could be negatively affected). ${ }^{3}$ However, altered mood states and reduced cognitive functioning are commonly reported following any disruption of normal sleeping behaviour. ${ }^{2,3,12}$ Nevertheless, these findings are based on lab settings, where performance and physiological measures were analysed isolated from complex sport activities.

Recent research underlines the importance of sleep for competition performance indicating there is a relationship between poor sleep quality and loss in a competition, ${ }^{13}$ and between sleep duration and competition performance, ${ }^{14}$ respectively. Accumulated evidence points at compromised sleep among the athletic population. $1,4,15$ While the National Sleep Foundation recommends 7 to 9 hours of sleep for young adults (18-25 years) as well as for adults (26-64 years), ${ }^{16}$ sleep durations of athletes are frequently below 7 hours. ${ }^{1}$ Lastella and colleagues ${ }^{4}$ assessed sleep-wake behaviours of 124 elite athletes across individual and team sports via actigraphy reporting 6.8 hours of sleep with an average sleep efficiency of $86 \%$, whereas athletes from individual sports obtained less sleep and poorer sleep efficiency $(6.5 \pm 1.1$ hours, $85.9 \pm 6.1 \%)$ than team sport athletes $(7.0 \pm 1.2$ hours, $86.4 \pm 4.8 \%)$. This finding is supported by a systematic review reporting that total sleep time is shorter in individual sports compared with team sports, and that sleep duration is shorter in female athletes. ${ }^{1}$ As an example, a recent questionnaire-based study showed that $50 \%$ of elite or highly-trained team sport athletes $(\mathrm{n}=$ 175) were identified as poor sleepers with the clinically significant prevalence of daytime sleepiness in $28 \%$ of the athletes. ${ }^{15}$ In addition, $38 \%$ showed signs of obstructive sleep apnoea, as they defined themselves as snorers, with $8 \%$ reporting apnoeic episodes. ${ }^{15}$ Moreover, more than three quarters of elite gymnasts $(n=67)$ reported abnormal scores in standardised sleep questionnaires (i.e., values above the clinical cut-off). ${ }^{14}$ Among 107 professional ice hockey players, $22 \%$ reported sleeping problem during off season, whereas $46 \%$ had compromised sleep during the competitive season. ${ }^{17}$ However, as most of these findings are based on subjective assessments, more clinical diagnoses of sleep disorders should be made available for the population of elite athletes to assess the interrelation between sleep and athletic as well as cognitive functioning.

\section{Challenges of sleep in sport}

Scheduling of training and competition, and time of season

Competition that is considered stressful in itself ${ }^{18,19}$ as well as the scheduling of competitions (e.g, late-night games $)^{20}$ are typical contributors to impaired sleep. Athletes often report worse sleep the night(s) before an important competition than at other times when competition is not imminent. ${ }^{18,19}$ Further, athletes report that they lack effective strategies to promote better sleep. ${ }^{18,19}$ While poor sleep before competitions is likely to be associated with anxiety, arousal and occupying thoughts, this may be addressed with psychological regulation techniques such as relaxation. The scheduling of competitions is not directly modifiable but can be anticipated with daytime naps (see below) or delayed recovery sessions on the following day.

Training schedules, especially early morning training, are also common factors that might lead to reduced sleep duration. ${ }^{21,22}$ Comparing rest days to training days, shorter sleep durations are 
obtained on (early-morning) training days due to the fact that bedtime cannot be advanced at will. $^{21,22}$ Moreover, training intensity and prolonged training stress have to be considered among challenges of sleep. ${ }^{23,24}$ Hausswirth and colleagues ${ }^{23}$ have shown that intensive overload training over three weeks in triathlon does not only lead to immunological changes but also to impaired sleep efficiency. Further, continuous intensive training over three months in preparation for a premiere led to disturbed sleep among ballet dancers. ${ }^{24}$

\section{Electronic devices}

The use of electronic devices before bedtime and after lights out has been shown to be negatively associated with sleep and daytime functioning. ${ }^{25,26}$ As an explanation for this, it has been suggested that the emission of blue light from electronic devices influences the circadian regulation of sleep promotion (due to an inhibition of the secretion of melatonin); however, stimulating and interactive social media platforms causing emotional arousal might also explain this phenomenon. ${ }^{27}$ Accordingly, volitional behaviour, i.e. self-regulation, is one of the underlying mechanisms behind this challenging factor and can be addressed with sleep hygiene strategies (see below).

\section{Travel and jet-lag}

Given the competition demands of most professional sports, regular short-haul (domestic) and long-haul (international) travel is a necessity. Whilst competition itself is a factor in disturbed sleep, the concomitant domestic or international travel associated with competition demands can further interfere with sleep behaviour. ${ }^{28}$ That is, as a result of the duration or extent of travel (e.g., changes in time-zones), a combination of jet-lag and/or travel fatigue can disrupt sleeping patterns. ${ }^{29}$ More specifically, sleep behaviour can be interrupted by the act of travel in that the seated and cramped conditions in a noisy and hypoxic environment can make the onset and maintenance of sleep difficult and thus result in travel fatigue. ${ }^{29}$ Alternatively, the rapid traversing of multiple time zones results in jet-lag due to desynchronization of circadian rhythms with external daylight events and is thus resulting in difficulty of sleeping or waking based on new local times. ${ }^{30}$ Either way, when athletes are required to travel to or from a competition, there is a risk of disrupted sleeping patterns; although most short-haul flights result in minimal disruption of sleep quantity or quality. ${ }^{31}$ Rather, long-haul or overnight travel is more likely to result in disrupted sleep behaviours and poorer sleep quantity and quality. ${ }^{32} \mathrm{~A}$ limited number of studies investigating the effects of jet-lag in athletes indicates indirect effects on performance as a consequence of changes in mood and arousal that are concurrent to reduced sleep (for a review see Duffield \& Fowler $^{28}$; Kölling et al. ${ }^{2}$ ). In terms of supporting recovery processes and enhancing an adaptation to the destination, behavioural strategies, such as arranging sleep-wake and meal times according to the target time, should be preferred over medications. ${ }^{30}$ The use of sleep medication will be further discussed in the subsequent section.

\section{Sleep interventions}

Behavioural strategies to promote sleep

While some of the challenging factors that have an external influence on sleep patterns cannot be easily removed or altered, the most obvious approach is education regarding lifestyle habits. Simple strategies, such as the implementation of consistent sleep-wake patterns and nonstimulating pre-bedtime routines, can be applied to improve individual sleep. ${ }^{27,33,34}$ Educating athletes about the role of sleep for athletic performance and the impact of detrimental behaviour (e.g., the use of electronic devices in bed and participating in social media after lights out) seem to be beneficial strategies. ${ }^{17,35}$ Moreover, red light in the evening might support secretion of the sleep regulating neurohormone melatonin, and thus promoting sleep onset as well as sleep quality. ${ }^{36}$ For example, Zaho et al. ${ }^{36}$ could show that a two-week treatment of whole-body red light therapy had a positive effect on subjective sleep ratings and changes in endurance 
performance as well as serum melatonin levels. The authors of that study assume that red light illumination may be related to increased arteriolar vasodilation and peripheral microcirculation. As sleep is further initiated through a reduced body core temperature which is achieved by elevating the distal skin temperature ${ }^{6}$ another pre-bedtime routine could be a warm shower to reduce sleep onset latency. ${ }^{37}$ The environmental temperature, on the other hand, should be preferably rather cool instead of warm.

\section{Sleep extension \& napping}

If the training or competition schedule provides sufficient space for recovery strategies, extending bedtime to achieve prolonged sleep duration appears a beneficial approach. Performance improvements have been reported for basketball players ${ }^{38}$ and tennis players ${ }^{39}$ after extending sleep duration for several weeks or nights, respectively. However, phases of extended sleep periods may only provide additional value if there is enough time to develop sleep pressure in the evening (i.e., a drive to sleep), otherwise initiating sleep can become difficult. ${ }^{40}$ Nevertheless, one night of extended sleep can improve the perceived recovery-stress state and mood in athletes. ${ }^{12}$ Overall, the number of (experimental) studies examining the effects of sleep extension on performance is still limited. As prevalence of sleep restriction among athletes seems to be quite high (as outlined above), athletes are encouraged to implement 30 to 60 minutes of additional sleep each night as a 'self-experiment' which should be monitored and supported by staff members. ${ }^{41}$

Athletes may also achieve additional sleep time by implementing daytime naps when feasible. Research regarding the efficacy of napping is limited, but it seems that beneficial effects can be detected following sleep restriction ${ }^{42}$ as well as following a 'normal' night of sleep. ${ }^{43}$ However, Petit and colleagues ${ }^{44}$ did not find positive effects of a 20-min nap on performance. On the other hand, they did not find negative effects on performance or on sleep architecture either. Thus, habitual napping can be generally encouraged, whereas timing (i.e., preferably in the early afternoon) and duration (i.e., $<30 \mathrm{~min}$ ) should be properly realised. ${ }^{45}$

\section{Medication}

Data on the use of sleep medication is basically limited to anecdotal reports, as it seems common to not only use prescription drugs but also self-administered over-the-counter medications. ${ }^{46}$ Typical circumstances where sleep medication is applied in the high-performance setting are (a) sedative action to reduce arousal, (b) chronobiological purposes to reduce jet-lag symptoms and (c) to manage insomnia in habitually poor sleepers. ${ }^{46}$ However, evidence for their effectiveness within healthy populations, especially athletes, is scarce, and little is known about their influence on subsequent daytime functioning and athletic performance. ${ }^{46}$ As some pharmaceuticals may have unknown side effects and could potentially cause dependency, athletes should be educated to prevent self-administration and off-label use of such medications. Effective sleep monitoring is, therefore, an important approach to detect sleep disorders and to manage 'healthy' sleep behaviour.

\section{Dreams and lucid dreaming to improve training}

Dreaming - or better sleep mentation - defined as all mental experiences (perceptions, thoughts, and emotion) occurring during sleep may provide another influence on recovery and performance in sport. Dreams usually reflect waking activities. For example, sport students who are frequently involved in sport activities during the day show higher active participation in sport or sport themes in dreams compared to psychology students. Especially under stressful situations, such as competitions, the dream content of athletes may be altered and consequently evoke disturbed dreaming. In a questionnaire study, approximately $15 \%$ of athletes $(n=840)$ stated that they experienced at least one distressing dream before an important competition or game during the preceding 12 months. Dream content might also mirror the need for recovery 
or recovery related processes, however, so far research has not examined dream content in athletes after high intensity exercise.

When the sleeping individual is aware of dreaming and capable of influencing the contents of the dream, a state of lucid dreaming is achieved. Experimental research demonstrated that lucid dream practice has a positive effect on waking performance. Recently, Stumbrys and colleagues instructed participants to practice a finger tapping task in their dreams and compared the pre to post-test performance change with a practical, mental and control group, respectively. The lucid dream practice group showed higher effects in finger tapping compared to mental imagery. However, this technique does not seem very common among athletes. More experimental research is required in terms of techniques to induce lucid dreaming and the effect of this on performance. On the other hand, as sleep supports the regeneration processes and aims to purposely detach athletes from their sport, lucid dreaming may interfere with this process. Therefore, athletes and staff should carefully schedule 'dream practice' separate from designated recovery nights or even encourage lucid dream activities which possibly improve recovery (e.g., relaxation).

\section{Implications \& concluding remarks}

While sleep can be considered as universal human requirement, its implementation is highly individual which is posing a challenging issue for some elite athletes. Considering that the need for sleep and the response to training stimuli as well as sleep loss are inter-individually different, practitioners should utilise an individual approach to sleep promotion interventions. ${ }^{47}$ A regular monitoring of sleep behaviour (objective as well as subjective assessment) and the assessment of training parameters and recovery-stress responses are therefore important in elite sports. ${ }^{48}$ On the one hand, this allows for detecting irregularities (e.g., due to travel or illness) and deriving interventions at an early stage. On the other hand, implementation of recovery modalities and sleep management strategies can be evaluated. Moreover, feedback of the data can be used to coach athletes in terms of the importance of regular sleep habits and increase the awareness of the mutual relationship between training stress and sufficient recovery. 


\section{References}

1. Gupta, L, Morgan, K, Gilchrist, S. Does elite sport degrade sleep quality? A systeamtic review. Sports Medicine. 2017;47:1317-1333. doi:10.1007/s40279-016-0650-6

2. Kölling, S, Ferrauti, A, Meyer, T, Pfeiffer, M, Kellmann, M. Sleep in Sports: A short summary of alterations in sleep/wake patterns and the effects of sleep loss and jet-lag. Deutsche Zeitschrift für Sportmedizin. 2016;67:35-38. doi:10.5960/dzsm.2016.215

3. Fullagar, HHK, Skorski, S, Duffield, R, Hammes, D, Coutts, AJ, Meyer, T. Sleep and athletic performance: The effects of sleep loss on exercise performance, and physiological and cognitive responses to exercise. Sports Medicine. 2015;45:161-186. doi:10.1007/s40279-014-0260-0

4. Lastella, M, Roach, GD, Halson, SL, Sargent, C. Sleep/wake behaviours of elite athletes from individual and team sports. European Journal of Sport Science. 2015;15:94-100. doi:10.1080/17461391.2014.932016

5. Dijk, D-J, Archer, SN. Light, sleep, and circadian rhythms: Together again. PLoS Biology. 2009;7:e1000145. doi:10.1371/journal.pbio.1000145

6. Kräuchi, K. The thermophysiological cascade leading to sleep initiation in relation to phase of entrainment. Sleep Medicine Reviews. 2007;11:439-451. doi:10.1016/j.smrv.2007.07.001

7. Carskadon, MA, Dement, WC. Monitoring and staging human sleep. In: Kryger, MH, Roth, T, Dement, WC eds. Principles and practice of sleep medicine. 5th ed. St. Louis: Elsevier Saunders; 2011:16-26.

8. Fuller, KL, Juliff, L, Gore, CJ, Pfeiffer, JJ, Halson, SL. Software thresholds alter the bias of actigraphy for monitoring sleep in team-sport athletes. Journal of Science and Medicine in Sport. in press. doi:10.1016/j.jsams.2016.11.021

9. Sargent, C, Lastella, M, Halson, SL, Roach, GD. The validity of activity monitors for measuring sleep in elite athletes. Journal of Science and Medicine in Sport. 2016;19:848-853. doi:10.1016/j.jsams.2015.12.007

10. Kölling, S, Endler, S, Ferrauti, A, Meyer, T, Kellmann, M. Comparing subjective with objective sleep parameters via multi-sensory actigraphy in German Physical Education students. Behavioral Sleep Medicine. 2016;14:389-405. doi:10.1080/15402002.2015.1017096

11. Walker, MP, Stickgold, R. It's practice, with sleep, that makes perfect: Implications of sleep-dependent learning and plasticity for skill performance. Clinics in Sports Medicine. 2005;24:301-317.

12. Kölling, S, Steinacker, JM, Endler, S, Ferrauti, A, Meyer, T, Kellmann, M. The longer the better: Sleep-wake patterns during preparation of the World Rowing Junior Championships. Chronobiology International. 2016;33:73-84. doi:10.3109/07420528.2015.1118384

13. Brandt, R, Bevilacqua, GG, Andrade, A. Perceived sleep quality, mood states, and their relationship with performance among Brazilian elite athletes during a competitive period. Journal of Strength Conditioning Research. 2016;31:1033-1039.

14. Silva, M-RG, Paiva, T. Poor precompetitive sleep habits, nutrients' deficiencies, inappropriate body composition and athletic performance in elite gymnasts. European Journal of Sport Science. 2016;16:726-735. doi:10.1080/17461391.2015.1103316

15. Swinbourne, R, Gill, N, Vaile, J, Smart, D. Prevalence of poor sleep quality, sleepiness and obstructive sleep apnoea risk factors in athletes. European Journal of Sport Science. 2016;16:850-858. doi:10.1080/17461391.2015.1120781

16. Hirshkowitz, M, Whiton, K, Albert, SM, Alessi, C, Bruni, O, DonCarlos, L, Hazen, N, Herman, J, Katz, ES, Kheirandish-Gozal, L, Neubauer, DN, O'Donnell, AE, Ohayon, M, Peever, J, Rawding, R, Sachdeva, RC, Setters, B, Vitiello, MV, Catesby Ware, J, Adamas Hillard, PJ. National Sleep Foundation's sleep time duration recommendations: 
Methodology and results summary. Sleep Health. 2015;1:40-43. doi:10.1016/j.sleh.2014.12.010

17. Tuomilehto, H, Vourinen, V-P, Penttilä, E, Kivimäki, M, Vourenmaa, M, Venojärvi, M, Airaksinen, O, Pihlajamäki, J. Sleep of professional athletes: Underexplored potential to improve health and performance. Journal of Sports Sciences. in press. doi:10.1080/02640414.2016.1184300

18. Erlacher, D, Ehrlenspiel, F, Adegbesan, OA, El-Din, HG. Sleep habits in German athletes before important competitions or games. Journal of Sports Sciences. 2011;29:859-866. doi:10.1080/02640414.2011.565782

19. Juliff, LE, Halson, SL, Pfeiffer, JJ. Understanding sleep disturbance in athletes prior to important competitions. Journal of Science and Medicine in Sport. 2015;18:13-18. doi:10.1016/j.jsams.2014.02.007

20. Fullagar, HHK, Skorski, S, Duffield, R, Julian, R, Bartlett, J, Meyer, T. Impaired sleep and recovery after night matches in elite football players. Journal of Sports Sciences. 2016;34:1333-1339. doi:10.1080/02640414.2015.1135249

21. Sargent, C, Lastella, M, Halson, SL, Roach, GD. The impact of training schedules on the sleep and fatigue of elite athletes. Chronobiology International. 2014;31:1160-1168. doi:10.3109/07420528.2014.957306

22. Sargent, C, Halson, SL, Roach, GD. Sleep or swim? Early-morning training severely restricts the amount of sleep obtained by elite swimmers. European Journal of Sport Science. 2014;14:S310-S315. doi:10.1080/17461391.2012.696711

23. Hausswirth, C, Louis, J, Aubry, A, Bonnet, G, Duffield, R, Le Meur, Y. Evidence of disturbed sleep and increased illness in overreached endurance athletes. Medicine and Science in Sports and Exercise. 2014;46:1036-1045. doi:10.1249/MSS.0000000000000177

24. Fietze, I, Strauch, J, Holzhausen, M, Glos, M, Theobald, C, Lehnkering, H, Penzel, T. Sleep quality in professional ballet dancers. Chronobiology International. 2009;26:1249-1262. doi:10.1080/07420520903221319

25. Exelmans, L, Van den Bulck, J. Bedtime mobile phone use and sleep in adults. Social Science \& Medicine. 2016;148:93-101. doi:10.1016/j.socscimed.2015.11.037

26. Saling, LL, Haire, M. Are you awake? Mobile phone use after lights out. Computers in Human Behavior. 2016;64:932-937. doi:10.1016/j.chb.2016.08.006

27. Caia, J, Kelly, VG, Halson, SL. The role of sleep in maximising performance in elite athletes. In: Kellmann, M, Beckmann, J eds. Sport, recovery and performance: Interdisciplinary insights. Abingdon: Routledge; 2018:151-167.

28. Duffield, R, Fowler, PM. Domestic and international travel: Implications for performance and reocvery in team-sport athletes. In: Kellmann, M, Beckmann, J eds. Sport, recovery and performance: Interdisciplinary insights. Abingdon: Routledge; 2018:183-197.

29. Waterhouse, J, Reilly, T, Edwards, B. The stress of travel. Journal of Sports Sciences. 2004;22:946-965. doi:10.1080/02640410400000264

30. Reilly, T, Atkinson, G, Edwards, B, Waterhouse, J, Åkerstedt, T, Davenne, D, Lemmer, B, Wirz-Justice, A. Coping with jet-lag: A position statement for the European College of Sport Science. European Journal of Sport Science. 2007;7:1-7. doi:10.1080/17461390701216823

31. Richmond, LK, Dawson, B, Stewart, G, Cormack, S, Hillman, DR, Eastwood, PR. The effect of interstate travel on the sleep patterns and performance of elite Australian Rules footballers. Journal of Science and Medicine in Sport. 2007;10:252-258. doi:10.1016/j.jsams.2007.03.002

32. Fowler, P, Duffield, R, Lu, D, Hickmans, JA, Tannath, JS. Effects of long-haul transmeridian travel on subjective jet-lag and self-reported sleep and upper respiratory 
symptoms in professional rugby league players. International Journal of Sports Physiology and Performance. 2016;11:876-884. doi:10.1123/ijspp.2015-0542

33. Irish, LA, Kline, CE, Gunn, HE, Buysse, DJ, Hall, MH. The role of sleep hygiene in promoting public health: A review of empirical evidence. Sleep Medicine Reviews. 2015;22:23-36. doi:10.1016/j.smrv.2014.10.001

34. Nédélec, M, Halson, SL, Delecroix, B, Abaidia, A-E, Ahmaidi, S, Dupont, G. Sleep hygiene and recovery strategies in elite soccer players. Sports Medicine. 2015. doi:10.1007/s40279-015-0377-9

35. Van Ryswyk, E, Weeks, R, Bandick, L, O'Keefe, M, Vakulin, A, Catcheside, P, Barger, L, Potter, A, Poulos, N, Wallace, J, Antic, NA. A novel sleep optimisation programme to improve athletes' well-being and performance. European Journal of Sport Science. 2017;17:144-151. doi:10.1080/17461391.2016.1221470

36. Zhao, J, Tian, Y, Nie, J, Xu, J, Liu, D. Red light and the sleep quality and endurance performance of Chinese female basketball players. Journal of Athletic Training. 2012;47:673-678. doi:10.4085/1062-6050-47.6.08

37. Whitworth-Turner, C, Di Michele, R, Muir, I, Gregson, W, Drust, B. A shower before bedtime may improve sleep onset latency of youth soccer players. European Journal of Sport Science. in press. doi:10.1080/17461391.2017.1346147

38. Mah, CD, Mah, KE, Kezirian, EJ, Dement, WC. The effects of sleep extension on the athletic performance on collegiate basketball players. Sleep. 2011;34:943-950.

39. Schwartz, J, Simon, RDJ. Sleep extension improves serving accuracy: A study with college varsity tennis players. Physiology \& Behavior. 2015;151:541-544. doi:10.1016/j.physbeh.2015.08.035

40. Kamdar, BB, Kaplan, KA, Keizirian, EJ, Dement, WC. The impact of extended sleep on daytime alertness, vigilance, and mood. Sleep Medicine. 2004;5:441-448. doi:10.1016/j.sleep.2004.05.003

41. Simpson, NS, Gibbs, EL, Matheson, GO. Optimizing sleep to maximize performance: Implications and recommendations for elite athletes. Scandinavian Journal of Medicine and Science in Sports. 2017;27:266-274. doi:10.1111/sms.12703

42. Waterhouse, J, Atkinson, G, Edwards, BJ, Reilly, T. The role of a short post-lunch nap in improving cognitive, motor, and sprint performance in participants with partial sleep deprivation. Journal of Sports Sciences. 2007;25:1557-1566.

43. Pelka, M, Kölling, S, Ferrauti, A, Meyer, T, Pfeiffer, M, Kellmann, M. Acute effects of psychological relaxation techniques between two physical tasks. Journal of Sports Sciences. 2017;35:216-223. doi:10.1080/02640414.2016.1161208

44. Petit, E, Mougin, F, Bourdin, H, Tio, G, Haffen, E. A 20-min nap in athletes changes subsequent sleep architecture but does not alter physical performance after normal sleep or 5-h phase advance conditions. European Journal of Applied Physiology. 2014;114:305-315. doi:10.1007/s00421-013-2776-7

45. Milner, CE, Cote, KA. Benefits of napping in healthy adults: Impact of nap length, time of day, age, and experience with napping. Journal of Sleep Research. 2009;18:272-281.

46. Taylor, L, Chrismas, BCR, Dascombe, B, Chamari, K, Fowler, PM. Sleep medication and athletic performance - The evidence for practioners and future research directions. Frontiers in Physiology. 2016;7:Article 83. doi:10.3389/fphys.2016.00083

47. Fullagar, HHK, Bartlett, JD. Time to wake up: Individualising the approach to sleep promotion interventions. British Journal of Sports Medicine. 2016;50:143-144. doi:10.1136/bjsports-2015-095759

48. Heidari, J, Kölling, S, Pelka, M, Kellmann, M. Monitoring the recovery-stress state in athletes. In: Kellmann, M, Beckmann, J eds. Sport, recovery and performance: Interdisciplinary insights. Abingdon: Routledge; 2018:3-18. 
